\title{
Lymphatic System
}

National Cancer Institute

\section{Source}

National Cancer Institute. Lymphatic System. NCI Thesaurus. Code C12746.

The anatomic system that includes the lymph nodes, lymphoid tissues in various org ans

(spleen, thymus, tonsils, gastrointestinal tract), and lymphatic vessels. It is part of the immune system that offers protection against infections. 